\title{
The meaning, the sense and the significance: Translating the science of mesenchymal stem cells into medicine
}

\author{
Paolo Bianco ${ }^{1,{ }^{\star}}, \mathrm{Xu} \mathrm{Cao}^{2}$, Paul S. Frenette ${ }^{3}$, Jeremy J. Mao ${ }^{4}$, Pamela G. Robey ${ }^{5}$, Paul J. \\ Simmons ${ }^{6}$, and Cun-Yu Wang ${ }^{7}$ \\ ${ }^{1}$ La Sapienza Universita di Roma, Rome, Italy \\ 2Johns Hopkins University, Baltimore, MD \\ ${ }^{3}$ Albert Einstein College of Medicine, New York, NY \\ ${ }^{4}$ Columbia University College of Dental Medicine, New York, NY \\ ${ }^{5}$ National Institutes of Health, Bethesda, MD \\ ${ }^{6}$ Mesoblast Limited, Melbourne, Australia \\ ${ }^{7}$ University of California - Los Angeles, Los Angeles, CA
}

\begin{abstract}
Mesenchymal stem cells ("MSCs") are the focus of intensive efforts worldwide, directed not only at elucidating their nature and unique properties, but also at developing cell-based therapies for a diverse range of diseases. More than three decades have passed since the original formulation of the concept (revolutionary at the time) that multiple connective tissues could emanate from a common progenitor/stem cell retained in the post-natal bone marrow. Despite the many important advances made since that time, significant ambiguities still plague the field as to the nature, identity, function, mode of isolation and experimental handling of "MSCs." These uncertainties have a major impact on their envisioned therapeutic use.
\end{abstract}

\section{Narrowing the focus on the stem cell}

Unbeknown to most readers of a copious literature dealing with both fundamental and clinical aspects of "MSC" biology, the current, widespread general concept of MSCs represents the modification (the distortion, we will argue herein) of a notion that is both classical, and solidly proven experimentally. This notion reads that the postnatal human (mammalian) bone marrow includes a subset of non-hematopoietic, stromal cells that are both multipotent and self-renewing. While the multipotency of bone marrow-derived "MSCs" has been known for long (summarized in Owen and Friedenstein ${ }^{1}$ ), their ability to self-renew has only recently been supported by the proper in vivo evidence (Box 1). 2,3 When transplanted in vivo, these cells generate a miniature bone organ with proper histology and architecture, in which hematopoiesis from the recipient animal becomes established. These cells are, or can be usefully conceptualized as, bona fide stem cells for all

*Corresponding Author: Paolo Bianco, M.D., Department of Molecular Medicine, Universita "La Sapienza”, Viale Regina Elena 324, Rome 00161, Italy, paolo.bianco@ uniroma1.it, Telephone: +39-06-444-1049. 
tissues that are found within a skeletal segment (a single bone): bone tissue itself, cartilage, adipocytes, fibroblasts, and hematopoiesis-supporting stroma. All are generated in vivo in proper transplantation assays; all are generated from a single cell, if a single cell is originally isolated and used to generate a transplantable clonal progeny (reviewed in Bianco et al. ${ }^{4}$ ). This concept, rooted in a line of experimentation originating in the 1960s, precisely defines the range of cell types that together comprise the progeny of the putative stem cell. It does so, based on the results of in vivo transplantation experiments that were progressively refined, and led stepwise to assigning a genuine, in vivo skeletogenic potential first to uncultured bone marrow tissue as a whole, ${ }^{5}$ then to its non-hematopoietic (stromal) cells, then to a subset of stromal cells capable of initiating clonal growth ex vivo (CFU-Fs), ${ }^{6}$ and ultimately to an in vivo identified perivascular (adventitial) cell amenable to prospective isolation based on phenotype (Box 1). ${ }^{2,3}$

\section{Box 1}

\section{What defines a (mesenchymal) stem cell: transplantation assays}

All definitions of stem cells are functional in nature, and all types of stem cells are defined by functional assays. These aim to prove their capacity to generate fully differentiated tissues in vivo-multipotency - and the ability to reconstitute in vivocells identical in phenotype and potency to those explanted-self-renewal. All these in vivoassays are simply the legacy (for each type of stem cells) of the seminal experiments that first revealed the defining functional properties of each type of stem cell. For example, functional identification of pluripotent (embryonic or reprogrammed) stem cells requires teratoma formation, generation of chimeras and germline transmission. Functional identification of hematopoietic stem cells requires long-term hematopoietic reconstitution through serial transplantation. The identification of defining functional properties through in vivoexperiments precedes, and is indispensable for, the identification of molecular markers of stem cells. Specifically intended to predict(with variable accuracy) the defining properties of stem cells (which are probed in vivo), markers can in turn only be identified through the use ofin vivoassays.

Generation of a heterotopic "ossicle" is the assay that defines 'MSCs' exactly like teratoma formation defines pluripotent cells, and hematopoietic reconstitution defines HSCs. Heterotopic transplantation aims to show the inherent ability of a given cell strain to generate histology-proven skeletal tissues, independent of exogenous, skeletogenic cues that would either be applied ex vivo, or come (in vivo) from grafting cells within a bone (orthotopic transplantation). The identification of multipotent, self-renewing bone marrow-derived 'MSCs' was specifically established by a sequential series of in vivoexperiments refined stepwise over a few decades (Fig. B1).

1. Transplantation of boneless, whole BM fragments results in the formation of heterotopic 'ossicles' (miniature bone organs that include the hematopoietic microenvironment), revealing the existence of a skeletogenic potential within $\mathrm{BM}$ and representing the seminal experiment that paves the way to identifying $\mathrm{MSCs}^{5}$. 
2. Generation of heterotopic ossicles by transplantation of stromal (adherent, nonhematopoietic) cell strains en lieu of tissue fragments ascribes the skeletogenic potential to the non-hematopoietic fraction of BM. In these experiments, conducted prior to the development of cell sorting, cell culture is in essence used to separate stromal from hematopoietic cells.

3. Generation of heterotopic ossicles by transplantation of cell strains from multiple CFU-Fs establishes the existence of in vivo assayable stromal progenitors in the bone marrow. Here, clonogenicity in vitro is used to define the stromal progenitors, by analogy with clonogenic hematopoietic progenitors able to generate colonies of hematopoietic cells in methyl-cellulose (ColonyForming Cells, CFC assays $)^{6}$.

4. Generation of heterotopic ossicles from single clones, grown from a single progenitor (CFU-F) proves that the individual clonogenic progenitor is a multipotent progenitors of skeletal tissues. All tissues in the ossicle (bone, adipocytes, fibroblasts) are derived from a single clonogenic progenitor, through its clonal progeny, which is generated in vitro and then transplanted in vivo. ${ }^{55}$

5. Formation of heterotopic ossicles by single progenitors which were explanted from the bone marrow based on a defined phenotype, followed by secondary passage and transplantation of identical cells harvested from the ossicle, establishes that multipotent progenitors can also self-renew. ${ }^{2,3}$

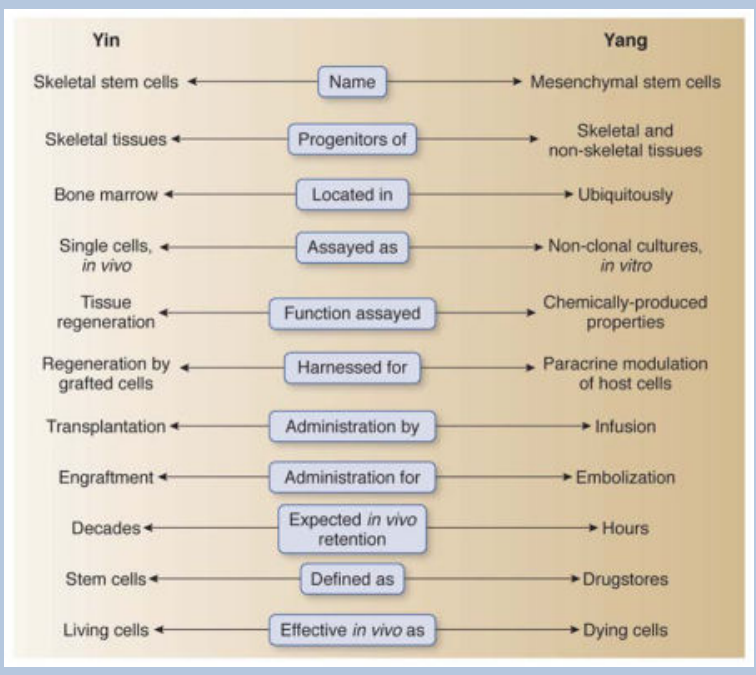

\section{What's in a name}

Whether called a "mesenchymal stem cell" as proposed by Caplan, ${ }^{7}$ and Pittenger et al., ${ }^{8}$ or more stringently (based on its proven in vivo differentiation potential) a skeletal stem cell, ${ }^{9}$ this cell is a precisely defined physical and conceptual entity.

Physically, this cell resides at the outer surface of sinusoids, a characteristic type of blood vessels in the bone marrow, in a subendothelial (mural) position (Fig. 1). It is part of a threedimensional perivascular stromal compartment in the bone marrow that originates (during 
organogenesis of bone) from committed osteogenic cells that reside at the primitive perichondrium and invade the bone anlage along with growing blood vessels (Fig. 2). ${ }^{4,10-14}$. A range of surface markers allow for prospective isolation of perisinusal MSCs (CD146, CD105, ALP, STRO-1, VCAM1 in humans; ${ }^{2}$; CD105, CD90, VCAM1 in the mouse (pending further refinement). ${ }^{15}$ Some of these can also be used as in situ markers in the intact bone marrow or in heterotopic transplants. The transcriptome of bone marrow MSCs combines expression of genes characteristic of committed early osteogenic cells (including the osteogenic master gene, Runx2) with genes characteristic of perivascular cells. ${ }^{2}$

Conceptually, an "MSC" is the single cell capable of generating a complete heterotopic bone/bone marrow organ (an "ossicle") in vivo, including a compartment of perivascular stromal cells with similar phenotype and properties as the originally explanted cell. ${ }^{2}$ This in vivo potential is system-restricted and cell-autonomous; i.e., it excludes the potential to generate non-skeletal tissues, and it does not require cueing with factors inducing a skeletogenic transcriptional program such as BMPs. Importantly, the self-renewing progenitor of skeletal tissues is at the same time the organizer of the hematopoietic microenvironment/niche in bone marrow. 2,3,16 Both arms of this dual function are of paramount physiological significance; only one arm (the capacity to generate structural tissue components of bone) has been the focus of significant translational attempts to date. Implications of the other arm, however, are huge: the "niche" function of MSCs, by itself, has the potential to lead into strategies for harnessing regulation of HSCs, or for modeling and targeting the interaction of hematopoietic and non-hematopoietic cancer with the bone environment.

\section{"As we may think"17}

As in other fields of stem cell biology, which underpin or complement successful clinical application, it was the use of fundamental tenets of stem cell biology at large $(i$, in vivo transplantation; $i i$, analysis at the single cell level) that allowed for the identification of a bona fide stem cell for skeletal tissues, and provided direct in vivo evidence for its translational potential. The progenitors of skeletal tissues that are directly proven to generate bone and other skeletal tissues in transplantation models were logically envisioned as fundamental tools for regeneration of bone in a number of clinical scenarios: first and foremost, for the repair of localized bone defects through local transplantation of either cells, or cell-material constructs that would restore bone integrity after trauma or extirpative surgery. ${ }^{18}$ In any organ system, however, the very notion of stem cells as fundamental postnatal units of organ or tissue regeneration modifies our understanding of organ physiology, and modifies the way in which individual diseases can be thought of, or modeled mechanistically. It also encourages, per se, visions of therapy even more daring than tissue engineering. For example, we now know that certain genetic skeletal diseases are best investigated, modeled in vivo, ${ }^{19}$ even genetically corrected in vitro, in skeletal stem cells; ${ }^{20}$ and our ability to identify and handle hematopoietic or epithelial progenitor/stem cells has already enabled correction through gene therapy in stem cells of lethal genetic diseases such as ADA-SCID,${ }^{21}$ or Epidermolysis Bullosa. ${ }^{22}$ Likewise, the notion of a stem cell that can be handled or modified ex vivo, and transplanted in vivo, would encourage attempts to tackle crippling or lethal diseases that affect the skeleton systemically, such as 
Osteogenesis Imperfecta (OI) or Fibrous Dysplasia. Here, insufficient consideration of the inherent specific biology of skeletal and connective tissues (their three-dimensional nature, slow turnover, matrix-centered cell cueing, and vascularity), and of their radical diversity in many functional and structural respects from blood or epithelia, has often resulted in hasty attempts towards translation. For example, early attempts to correct OI by bone marrow transplantation, ${ }^{23-25}$ based on the assumption that bone marrow skeletal progenitors would engraft to bone as efficiently as hematopoietic stem cells (HSCs) do, were naïve insofar as oblivious to the specific biology of skeletal progenitors - which do not engraft into the skeleton via the circulation. Simple or complex, immediate prospects or wishful thinking, all these approaches are logically linked in a simple and straightforward way to the biological concept behind them, and to the clear-cut experimental evidence that led to the formulation of the concept: as we learn that a stem cell for skeletal tissues exists, we next try to harness its proven physiological function. This simple conceptual framework (organ-specific stem cells for organ-specific diseases), not surprisingly, is the one in which all instances of clinically successful translation (in one case, with epochal impact) of stem cell science in medicine known to date are inscribed: bone marrow transplantation, ${ }^{26}$ regeneration of skin, ${ }^{27-29}$ and regeneration of cornea. ${ }^{3031}$

\section{The clash of two concepts}

The fundamental science to be harnessed in this conceptual framework, however, is precisely sculpted: it portrays committed multipotent progenitors for skeletal tissues, and skeletal tissues only; it defines them as residing within the bone marrow, and not elsewhere or everywhere; it defines them as locally transplantable, but not necessarily as systemically transplantable. This precise notion, and all the prior science behind it, was imprecisely used in the 1990s to formulate a quite different concept. ${ }^{7}$ This different concept was to become popular and irreversibly linked to the term "mesenchymal stem cells," which has now attained universal use. The "revisionist" concept departs from the original notion in two important respects: first, it postulates a common progenitor not just of skeletal tissues, but of all non-epithelial, non-hematopoietic tissues derived from mesoderm (that is, including skeletal muscle, myocardium, endothelium etc., seen as part of a hypothetical "mesengenic process" 32 that does not match established knowledge in developmental biology); ${ }^{33}$ second, it assumes that such progenitors are not found in the bone marrow only, but also in a number of other tissues, virtually in all postnatal organs. ${ }^{34}$ Neither point is proven. Whereas the original concept of skeletal stem cells was (and remained) rooted in stringent in vivo transplantation and single cell assays, the revisionist concept is complemented by nonstringent in vitro assays and does not make use of single cell analysis.

\section{An awkward position}

Crystallized in a widely cited "position paper" that assumed to set criteria defining "MSCs" for general use, ${ }^{35}$ the view finally became dominant that every non-clonal culture of cells from any connective tissue, expressing a limited range of non-specific surface markers in vitro, and amenable under artificial chemical cueing to artificial accumulation of hydrophobic, mineralized, or polyanionic material (liberally interpreted as fat, bone, and cartilage, respectively), would represent a culture of MSCs capable of generating, 
respectively, adipocytes, osteoblasts and chondrocytes - efficiently, robustly, and in vivo, as expected from stem cells in a translational perspective. Based on this loose set of criteria, indeed "MSCs" can be grown in culture from virtually every tissue. Not validated by proper in vivo and clonal assays (Box 2), the artificial properties seen as "defining" features of "MSCs" are simply widely shared properties of connective tissue cells, without implying any true stem cell property, or the true ability to form bone, cartilage or adipose tissue in vivo. The confusion introduced by these criteria has major consequences when "translational" implications are considered. If predicted based on unreliable in vitro criteria, all properties of the "MSC" to be harnessed in patients (such as, in the simplest scenario, the ability to regenerate bone) are predicted unreliably: placenta, menstrual endometrium, and bone become conceivably equivalent sources of cells for efficient bone regeneration, while they absolutely are not. Or else, one could conceive a clinical trial to treat Duchenne's muscular dystrophy (a lethal disease) with fat-derived MSCs, claimed to be more efficiently myogenic than genuine myoblasts. ${ }^{36}$ More in general, as the range of clinical conditions seen as potentially treatable by systemically administered "MSCs" expands, and relevant clinical trials are initiated, it is the mere intravenous infusion of nondescript cultured connective tissue cells in patients that becomes confused with, but proposed and presented as, a stem cell-based therapy.

\section{Box 2}

\section{What can you determine from a culture of BM stromal cells}

Cultures of bone marrow stromal cell populations (improperly referred to as cultures of mesenchymal stem cells) can be established through two different procedures.

The conventional method involves the direct establishment of cultures from total marrow cell suspensions and is based on isolation by plastic adherence (Fig. 1). If cell suspensions are plated in bulk (non-clonal density), monolayers of non-hematopoietic, adherent cells are generated, representing cultures of total bone marrow stroma, not of stem cells. Both stromal progenitors (clonogenic cells, CFU-F) and stromal cells that are neither stem cells nor progenitors, but are capable of limited growth, contribute to these cultures. These cultures cannot be used for assays of multipotency. By plating the cell suspension at clonal density, multiple stromal colonies are generated, where each is the clonal progeny of a single stromal progenitor/stem cell (CFU-F). When all colonies are passaged together and further cultured, the resulting cell strain is the progeny of multiple clonogenic stromal progenitors, which typically may differ from one another in differentiation potential. These cultures, again, cannot be used to assess multipotency. When a single colonyis harvested and cultured, the resulting cell strain is the clonal progeny of a single stromal progenitor. Thesecultures cantherefore be used to assess multipotency. Pending the development of methods for transplanting a single, unculturedprogenitor (which must necessarily be identified by phenotype), in vivo transplantation of the clonal progeny of a single clonogenic progenitor is the standard assay for probing (proving) multipotency. Growing clones of murine MSCs in vitro is more problematic than growing clones of human MSCs, and it imposes specific technical hurdles, which may be circumvented in different ways ${ }^{3,63}$. 
An alternative method of isolation is based on surface phenotype, so-called 'prospective' isolation (Fig. 1). This necessarily involves the use of one or more markers (STRO-1, CD146, CD105, ALP, CD49a, CD271, among others, in humans, and nestin, CD105, VCAM1, CD90 and others in mice) for cell sorting. Plating the sorted population at clonal density results in the establishment of progenitor (CFU-F)-initiated colonies; the frequency and relative representation of CFU-Fs (stromal progenitors) within the phenotype-defined population, are estimated by colony forming efficiency (CFE) assays. A purified population of progenitorswould have a colony forming efficiency of $100 \%$ at clonal density $\left(\leq .6 \mathrm{cells} / \mathrm{cm}^{2}\right)$. A purified population of stem cellswould be comprised of cells that would be uniformly multipotent, and uniformly self-renewing, as assessed by in vivo transplantation.

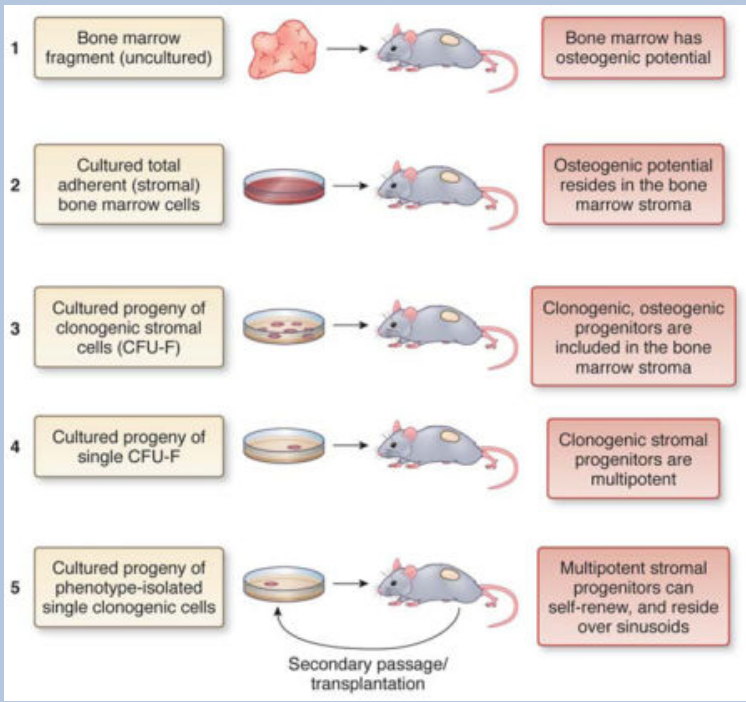

\section{Wrapped around your vessel}

The demonstration that in human bone marrow, "MSCs" are perivascular cells ${ }^{2}$ (an observation later reproduced in the mouse as well ${ }^{3}$ ) led some to contend that "pericytes" and MSCs are coinciding entities. Combined with the speculation that MSCs are ubiquitous, this idea would conveniently identify pericytes as the ubiquitous in situ counterpart of the ubiquitous MSCs. ${ }^{36,37}$ Pericytes are perivascular cells found in all tissues; they are not a distinct lineage; they are recruited to blood vessel walls from the surrounding tissue, during organ development and growth (Fig. 2) ${ }^{38-40}$ The idea that distinct classes of lineagerestricted, tissue-specific progenitors, would in the same way and through the same mechanisms (PDGF-BB/PDGFR $\beta$ loop, Ang-1, TGF $\beta 1$ ) be recruited to (and stabilized within) microvessel walls from the local environment in developing and growing tissues was recently proposed. ${ }^{2}$ This idea would explain why skeletal progenitors are perivascular in the bone marrow; why some perivascular cells in skeletal muscle are myogenic; ${ }^{41}$ and why adipocyte progenitors in adipose tissue are perivascular as well. ${ }^{42}$ Far from being consistent with the opposite idea that the ubiquitous pericytes coincide with the ubiquitous multilineage MSCs,${ }^{36,43}$ these data suggest that specific classes of tissue progenitors with specific differentiation capacities can be isolated from different tissues. ${ }^{4}$ The perivascular position of 
these cells in different tissues, reflected in the shared expression of adhesion molecules mediating the interaction with endothelial cells through homophilic interaction (MCAM/ CD146) and surface receptors/antigens involved in regulation of perivascular cells (e.g., PDGF-R $\beta / C D 140 b$; CD105/endoglin) could provide a lead into efficient prospective isolation. To prove this, identical conditions for cell isolation, culture, and in vivo transplantation in rigorous assays must be defined and used. These studies might provide significant advances as to how to identify and isolate specific classes of tissue progenitors for specific regenerative applications in specific tissues and organs.

\section{Plasticity melts}

During the past decade, a number of studies have pursued the idea that a broad range of extra-skeletal tissues could be directly regenerated by "MSCs" Notably, these tissues included not only extra-skeletal tissues that are otherwise derived from mesoderm (as are skeletal tissues, except the facial bones), but also derivatives of other germ layers such as liver cells or neurons. ${ }^{44}$ Inscribed in a broader trend claiming transgermal "plasticity" of somatic postnatal stem cells, the idea was entertained or implied for the last decade that MSCs or subsets thereof could be pluripotent cells, like embryonic stem cells (ESCs). ${ }^{45} \mathrm{~A}$ number of in vitro studies claimed efficient differentiation of chemically cued "MSCs" into disparate non-skeletal cell types, as a prelude to more daring preclinical and clinical studies. Less than rigorous criteria for assessing differentiation in artificial in vitro systems, and more than empirical ways of altering a cultured cell phenotype (a serendipitous, empirical, partial reprogramming ante litteram) were a unifying thread for virtually all of these studies. These studies drifted away from the principle that the system-specificity of post-natal stem cells (e.g., hematopoietic, epithelial) is cell-autonomous and not cued; that the general paradigm of in vitro cueing is well suited to explore technologies for directing differentiation of pluripotent ESCs, but not equally sound if transferred to committed postnatal progenitors; and that as applied to committed post-natal cells, exogenous cueing of directed differentiation is best seen as reprogramming (change of potential) rather than differentiation (expression of potential). As blatant examples, C2C12 myoblasts or endothelial cells can indeed be reprogrammed to an osteogenic phenotype by BMP-2/SMAD signaling, ${ }^{46,47}$ which induces the master transcription factors regulating osteogenesis, Runx2 and Osx/SP7 (the latter of which indeed was discovered exactly in BMP-treated C2C12 cells ${ }^{48}$ ). Yet, non-cued $\mathrm{C} 2 \mathrm{C} 12$ cells or endothelial cells do not spontaneously form bone if transplanted in vivo, which is what skeletal progenitors do. Likewise, induction of liver celllike phenotypic traits in marrow stromal cells ${ }^{45,49}$ does not mean either an inherent nature of stromal cells as liver cell progenitors, or that true liver cells can be obtained in this fashion with the efficiency and robustness that any conceivable translational purpose would imply. Enthusiasm and applicative motivations (hypes and hopes) often prevailed over caution and rigor, with some unfortunate pitfalls. In this way, for example, the reversible effects of agonists of adenylyl cyclase on F-actin and cell shape, long and widely known, ${ }^{50}$ were freely interpreted in several studies as the induction of a neuron-like phenotype in bone cells, ${ }^{44}$ while merely representing transient cytoskeletal disruption. 


\section{Can do, can't do}

The obviously erroneous idea that MSCs are pluripotent is now on the wane. Just as the echo of the isolation in culture of human embryonic pluripotent stem cells had cast on the mind of many the seductive suggestion that all cells called "stem cells" could be pluripotent, the echo of evidence that somatic cells (notably, including "MSCs" 51 ) need, in fact, to be "reprogrammed" (via defined protocols of transduction with defined sets of transcription factors ${ }^{52}$, in order to become pluripotent has now put the idea to rest. Nonetheless, a number of preclinical and clinical studies were planned and conducted over the last ten years aiming at regenerating the infarcted heart or brain, using cells whose ability to regenerate myocardium or brain was simply never proven. Almost invariably, these studies recorded some kind of effect that was defined as beneficial; actual differentiation into relevant cell types in vivo was claimed in early studies, but not confirmed in later ones. The notion that MSCs could regenerate the infarcted heart or the brain is no longer seriously entertained or defended; yet the notion that MSCs can nonetheless be beneficial for the infarcted heart or brain still is, and a number of clinical trials worldwide pursue evidence of those effects marking, anew, a true divorce of basic and translational science. At this time, a popular line of thinking contends that MSCs, while not endowed with properties of pluripotent cells, and thus unable to regenerate the target, non-skeletal tissues, still can exert beneficial effects of clinical relevance on a number of extra-skeletal organs through mechanisms unrelated to a progenitor function. ${ }^{53,54}$

\section{As a progenitor, or not as a progenitor}

The idea that "MSCs" can exert functions other than those characteristic of a stem/ progenitor cell (tissue regeneration), per se, is conceptually intriguing, and certainly supported by direct evidence. One defining feature of bone marrow-derived MSCs is indeed to establish, organize and transfer the hematopoietic microenvironment/niche in vivo. ${ }^{2,16,55}$ In in vivo systems that can recapitulate the ontogeny of bone and marrow, this function is intertwined with the organization of nascent blood vessels into functional networks, which appears to be directed by MSCs via direct interaction with endothelial cells. ${ }^{2}$ In other experimental systems, technically not designed or suited to allow for the skeletogenic potential of MSCs to unfold, their ability to organize vascular networks can even be portrayed in isolation; i.e., in the absence of bone or bone marrow. ${ }^{56-58}$ Thus, at least with respect to hematopoietic cells including hematopoietic stem cells, and with respect to endothelial cells, MSCs do exert a function that is not immediately traceable to a general stem cell property. Indeed, the ability of transplanted MSCs to act at the same time as progenitors of skeletal tissues (of donor origin), and as organizer of recipient tissues, is unique among all cells that are called stem cells. However, these non-progenitor functions of MSCs are, so far: a) restricted to bone marrow-derived MSCs, and b) only documented when MSCs are locally, not systemically, transplanted and engrafted. Of note, while only partially understood, mechanisms behind these properties of MSCs are not shrouded in obscurity or vagueness: their interaction with endothelial and hematopoietic cells is mediated by known factors such as FGF-2, PDGF-BB, TGF $\beta 1$, Angiopoietin-1, CXCL-12, and by their specific role as either producers of, or responders to such factors. ${ }^{2,38}$ Such a role for bone marrow-derived MSCs in these function is coherent with established functions 
of these factors themselves, in vascular and hematopoietic cell biology. While still not at hand, then, a general picture of how bone marrow-derived MSCs can regulate important physiological functions such as hematopoiesis, hematopoietic stem cell physiology, and assembly and remodeling of vascular lattices, can be reasonably expected to be completed in a foreseeable future. Indeed, recognizing the role of MSCs in these processes, and dissecting that role further, does in fact highlight innovative avenues for translation. These functions, at variance with others, are reasonably defined, can be reproducibly modeled in vivo, and can thus be dissected to the measure required to envision a clinical use, albeit future and not immediate. The role of "MSCs" (specifically, bone marrow-derived MSCs) as potential key contributors to the "niche" effect that characterizes HSC biology, for example, has obvious major implications for devising strategies for expansion of HSCs, a remaining major hurdle in clinical hematology. ${ }^{59}$. Likewise, learning how to harness the vessel-remodeling functions of MSCs might promise to improve tissue vascularity in different scenarios.

\section{Lost in translation}

In sharp contrast, other functions of MSCs that are evoked as a reason to move fast from confused, vague, or insufficient science to translation or therapy, are not clearly defined. Variably referred to as trophic, anti-inflammatory, or immune modulatory, these effects, seen from a distance, do evoke at least the flavor of the type of regulatory function that MSCs exert in their physical existence as bone marrow stromal cells. With some measure of imagination and none of science, one could relate regulation of immune and inflammatory cells at least to regulation of cells of hematopoietic lineage. However, some of the nonprogenitor effects of "MSCs" (such as immune modulation as predicted by inhibition of the mixed lymphocyte reaction), are not effect of stem cells, as they are duplicated in cultures of fibroblasts. ${ }^{60}$ Furthermore, the precise identity of such regulatory functions (and as an unavoidable consequence, of their mechanisms) remains, at present, unknown and unverified at best. Both the effects and the mechanisms escape validation in defined in vivo model systems to date. All regulatory functions of MSCs (of any cell) are expected to be in turn tightly regulated, and to occur in defined contexts of tissue physiology; understanding this regulation and these contexts would be crucial for their wished-for harnessing. Inflammation and abatement thereof, for example, occur sequentially in tissues, and involve significant, sequential modulation of function of individual cell types. Wound healing and tissue regeneration, likewise, do not coincide with one another (a scar heals a wound, but is not a regenerated organ). Presumed roles of "MSCs" in each type of process are often confused with one another. When inscribed in a "translational" perspective, necessary distinctions are further blurred: "paracrine" effects are said to operate in "injured" organs, regardless of the nature of the injury, which, of course, generates very different local milieus, microenvironments and patterns of cell response. Whether inflammatory (Crohn's disease), infectious (sepsis), ischemic (myocardial infarction, stroke), metabolic (blood glucose level control) or degenerative (Parkinson's disease) in nature, it would seem that all diseases ("injuries") of all organs would benefit from MSCs, ${ }^{54}$ which evokes a modern resurrection of alchemy. Based on these assumptions, MSCs are intravenously infused into patients with heart attacks, renal failure, stroke, diabetes, Crohn's disease, or Parkinson's disease; they are envisioned or proposed as treatment of autism or Huntington's disease, and 
dozens of other unrelated conditions that seem unlikely to represent reasonable targets being reasonably aimed at.

These considerations notwithstanding, the extent to which the purported "paracrine" beneficial effects of MSCs in all these conditions truly represent an indispensable role of cells (which could for example regulate other cells via cell-cell contacts, but would require engraftment to do so), rather than the role of one or more released factors, cannot be addressed in the absence of defined and reproducible models. In addition, dispensable or indispensable as a role of cells can be, it would remain unclear whether that role would be a role of stem cells, of cells, of cultured cells, or of cultured cells injected and impacted in blood vessels. Furthermore, this question cannot, by definition, be addressed by clinical trials, particularly when conducted in the absence of a reasonable understanding of pharmacokinetics of the administered "drug" (cells) and a solid rationale. Intravenously injected MSCs embolize in the lungs, where they cause endothelial damage, ${ }^{61,62}$, which per se would be a non-negligible, general adverse effect; they are then cleared in a matter of hours. Therefore, whatever paracrine effect they can exert, on whatever pathological process, must be short-lived and may be cell death-related. Clearly, intravenous infusion of cells that do not engraft, cannot be defined as transplantation. Effects of infused cells that are neither transplanted, nor engrafted, and do not regenerate tissues, per se do not reflect a stem cell or regenerative function, and their pursuit should not be presented as a stem cell based, or regenerative, therapy. The issue is not trivial, and is not intended to discount the role of empiricism in discovery of medicines. In all the history of Medicine, with all kinds of medicines (cell products) ultimately identified in either the vegetal or animal kingdom (digitalis or steroids, morphine or insulin, just to name the simplest), recognition of an empirically identified biological effect led to isolation (or synthesis) and use of the active principle and understanding of its mode of action. If indeed "MSCs" were affecting tissue injury and repair via paracrine factors as commonly claimed, then the factors, not the cells, would be the medicine. Then, intravenous injections of cells in patients, as opposed to the pursuit of the factors and their mechanisms at the bench, would turn into the opposite of a true translational approach. It is only sense, what one can translate.

\section{Looking ahead}

It is quite odd that precisely at the time when MSCs evolve from an experimentally-derived hypothesis into a recognizable entity, translational approaches centered on MSCs seem to drift away from the hypothesis, from the recognizable entity, and from the general paradigm that links stem cell properties to regenerative medicine. Verified by current data, the potential of the original concept (which first highlighted the functional continuity, encrypted in a single cell, of a single system made of bone and bone marrow), effectively fertilizes both basic science and translational opportunities in multiple, and at times new, directions. The role of MSC as niche cells, as tissue organizers, and as direct skeletal progenitors, each opens opportunities and poses challenges. Grasping the unique nature of the system and of the specific stem cell is necessary to identify how to harness science for therapy, which may involve entirely novel ways and a broad range of intermediate steps. Far from being reduced to the empirical injection of poorly characterized cultured cell strains, these include the stem cell-based modeling of disease mechanisms; the identification of cell-derived bioactive 
factors and their use as drugs; the definition of drug-targetable disease mechanisms operating specifically in the stem cells or the broad system of interrelated tissues they rule; the identification of strategies for cell delivery that take into account the specificities of the system and would permit the design of gene therapy strategies. Case histories of success in regenerative medicine reveal century-long scientific backgrounds. MSCs are truly the newcomers to the club, or maybe the founders of a new one. Give them time (and serious effort).

\section{References}

1. Owen M, Friedenstein AJ. Stromal stem cells: marrow-derived osteogenic precursors. Ciba Found Symp. 1988; 136:42-60. [PubMed: 3068016]

2. Sacchetti B, et al. Self-Renewing Osteoprogenitors in Bone Marrow Sinusoids Can Organize a Hematopoietic Microenvironment. Cell. 2007; 131:324-336. [PubMed: 17956733]

3. Mendez-Ferrer $S$, et al. Mesenchymal and haematopoietic stem cells form a unique bone marrow niche. Nature. 2010; 466:829-834. [PubMed: 20703299]

4. Bianco P, Robey PG, Simmons PJ. Mesenchymal stem cells: revisiting history, concepts, and assays. Cell Stem Cell. 2008; 2:313-319. [PubMed: 18397751]

5. Tavassoli M, Crosby WH. Transplantation of marrow to extramedullary sites. Science. 1968; 161:54-56. [PubMed: 4871792]

6. Friedenstein AJ, Chailakhjan RK, Lalykina KS. The development of fibroblast colonies in monolayer cultures of guinea- pig bone marrow and spleen cells. Cell Tissue Kinet. 1970; 3:393403. [PubMed: 5523063]

7. Caplan AI. Mesenchymal stem cells. J Orthop Res. 1991; 9:641-650. [PubMed: 1870029]

8. Pittenger MF, et al. Multilineage potential of adult human mesenchymal stem cells. Science. 1999; 284:143-147. [PubMed: 10102814]

9. Bianco, P.; Robey, PG. Skeletal stem cells. In: Lanza, RP., editor. Handbook of Adult and Fetal Stem Cells. Academic Press; San Diego: 2004. p. 415-424.

10. Streeter GL. Developmental horizons in human embryos: review of the histogenesis of cartilage and bone. Contr Embryol Carneg Inst. 1949; 33:149-169.

11. Bianco P, Bradbeer JN, Riminucci M, Boyde A. Marrow stromal (Western-Bainton) cells: identification, morphometry, confocal imaging and changes in disease. Bone. 1993; 14:315-320. [PubMed: 8363874]

12. Bianco P, Riminucci M, Kuznetsov S, Robey PG. Multipotential cells in the bone marrow stroma: regulation in the context of organ physiology. Crit Rev Eukaryot Gene Expr. 1999; 9:159-173. [PubMed: 10445154]

13. Arai F, Ohneda O, Miyamoto T, Zhang XQ, Suda T. Mesenchymal stem cells in perichondrium express activated leukocyte cell adhesion molecule and participate in bone marrow formation. $\mathrm{J}$ Exp Med. 2002; 195:1549-1563. [PubMed: 12070283]

14. Maes C, et al. Osteoblast precursors, but not mature osteoblasts, move into developing and fractured bones along with invading blood vessels. Dev Cell. 2010; 19:329-344. [PubMed: 20708594]

15. Chan CK, et al. Endochondral ossification is required for haematopoietic stem-cell niche formation. Nature. 2009; 457:490-494. [PubMed: 19078959]

16. Bianco P. Bone and the hematopoietic niche: a tale of two stem cells. Blood. 2011; 117:52815288. [PubMed: 21406722]

17. Bush V. As we may think. The Atlantic. 1945

18. Bianco P, Robey PG. Stem cells in tissue engineering. Nature. 2001; 414:118-121. [PubMed: 11689957]

19. Bianco P, et al. Reproduction of human fibrous dysplasia of bone in immunocompromised mice by transplanted mosaics of normal and Gsalpha-mutated skeletal progenitor cells. J Clin Invest. 1998; 101:1737-1744. [PubMed: 9541505] 
20. Piersanti S, et al. Transfer, analysis, and reversion of the fibrous dysplasia cellular phenotype in human skeletal progenitors. J Bone Miner Res. 2010; 25:1103-1116. [PubMed: 19874199]

21. Aiuti A, et al. Correction of ADA-SCID by stem cell gene therapy combined with nonmyeloablative conditioning. Science. 2002; 296:2410-2413. [PubMed: 12089448]

22. Mavilio F, et al. Correction of junctional epidermolysis bullosa by transplantation of genetically modified epidermal stem cells. Nat Med. 2006; 12:1397-1402. [PubMed: 17115047]

23. Horwitz EM, et al. Transplantability and therapeutic effects of bone marrow-derived mesenchymal cells in children with osteogenesis imperfecta. Nat Med. 1999; 5:309-313. [PubMed: 10086387]

24. Horwitz EM, et al. Clinical responses to bone marrow transplantation in children with severe osteogenesis imperfecta. Blood. 2001; 97:1227-1231. [PubMed: 11222364]

25. Horwitz EM, et al. Isolated allogeneic bone marrow-derived mesenchymal cells engraft and stimulate growth in children with osteogenesis imperfecta: Implications for cell therapy of bone. Proc Natl Acad Sci U S A. 2002; 99:8932-8937. [PubMed: 12084934]

26. Thomas ED, Lochte HL Jr, Lu WC, Ferrebee JW. Intravenous infusion of bone marrow in patients receiving radiation chemotherapy. N Engl J Med. 1957; 257:491-496. [PubMed: 13464965]

27. Green H. Regeneration of the skin after grafting of epidermal cultures. Lab Invest. 1989; 60:583584. [PubMed: 2716280]

28. De Luca M, Pellegrini G, Green H. Regeneration of squamous epithelia from stem cells of cultured grafts. Regen Med. 2006; 1:45-57. [PubMed: 17465819]

29. Ronfard V, Rives JM, Neveux Y, Carsin H, Barrandon Y. Long-term regeneration of human epidermis on third degree burns transplanted with autologous cultured epithelium grown on a fibrin matrix. Transplantation. 2000; 70:1588-1598. [PubMed: 11152220]

30. Pellegrini G, et al. Long-term restoration of damaged corneal surfaces with autologous cultivated corneal epithelium. Lancet. 1997; 349:990-993. [PubMed: 9100626]

31. Rama P, et al. Limbal stem-cell therapy and long-term corneal regeneration. N Engl J Med. 2010; 363:147-155. [PubMed: 20573916]

32. Caplan AI. The mesengenic process. Clin Plast Surg. 1994; 21:429-435. [PubMed: 7924141]

33. Tam PP, Trainor PA. Specification and segmentation of the paraxial mesoderm. Anat Embryol (Berl). 1994; 189:275-305. [PubMed: 8074321]

34. da Silva Meirelles L, Chagastelles PC, Nardi NB. Mesenchymal stem cells reside in virtually all post-natal organs and tissues. J Cell Sci. 2006; 119:2204-2213. [PubMed: 16684817]

35. Dominici M, et al. Minimal criteria for defining multipotent mesenchymal stromal cells. The International Society for Cellular Therapy position statement. Cytotherapy. 2006; 8:315-317. [PubMed: 16923606]

36. Crisan M, et al. A perivascular origin for mesenchymal stem cells in multiple human organs. Cell Stem Cell. 2008; 3:301-313. [PubMed: 18786417]

37. Caplan AI. All MSCs are pericytes? Cell Stem Cell. 2008; 3:229-230. [PubMed: 18786406]

38. Jain RK. Molecular regulation of vessel maturation. Nat Med. 2003; 9:685-693. [PubMed: 12778167]

39. Diaz-Flores L, et al. Pericytes. Morphofunction, interactions and pathology in a quiescent and activated mesenchymal cell niche. Histol Histopathol. 2009; 24:909-969. [PubMed: 19475537]

40. Armulik A, Genove G, Betsholtz C. Pericytes: developmental, physiological, and pathological perspectives, problems, and promises. Dev Cell. 2011; 21:193-215. [PubMed: 21839917]

41. Dellavalle A, et al. Pericytes of human skeletal muscle are myogenic precursors distinct from satellite cells. Nat Cell Biol. 2007; 9:255-267. [PubMed: 17293855]

42. Tang W, et al. White fat progenitor cells reside in the adipose vasculature. Science. 2008; 322:583-586. [PubMed: 18801968]

43. da Silva Meirelles L, Caplan AI, Nardi NB. In search of the in vivo identity of mesenchymal stem cells. Stem Cells. 2008; 26:2287-2299. [PubMed: 18566331]

44. Woodbury D, Schwarz EJ, Prockop DJ, Black IB. Adult rat and human bone marrow stromal cells differentiate into neurons. J Neurosci Res. 2000; 61:364-370. [PubMed: 10931522]

45. Jiang Y, et al. Pluripotency of mesenchymal stem cells derived from adult marrow. Nature. 2002; 418:41-49. [PubMed: 12077603] 
46. Katagiri T, et al. Bone morphogenetic protein-2 converts the differentiation pathway of $\mathrm{C} 2 \mathrm{C} 12$ myoblasts into the osteoblast lineage. J Cell Biol. 1994; 127:1755-1766. [PubMed: 7798324]

47. Medici D, et al. Conversion of vascular endothelial cells into multipotent stem-like cells. Nat Med. 2010; 16:1400-1406. [PubMed: 21102460]

48. Nakashima K, et al. The novel zinc finger-containing transcription factor osterix is required for osteoblast differentiation and bone formation. Cell. 2002; 108:17-29. [PubMed: 11792318]

49. Lee KD, et al. In vitro hepatic differentiation of human mesenchymal stem cells. Hepatology. 2004; 40:1275-1284. [PubMed: 15562440]

50. Willingham MC, Pastan I. Cyclic amp and cell morphology in cultured fibroblasts. Effects on cell shape, microfilament and microtubule distribution, and orientation to substratum. J Cell Biol. 1975; 67:146-159. [PubMed: 51851]

51. Niibe K, et al. Purified mesenchymal stem cells are an efficient source for iPS cell induction. PLoS One. 2011; 6:e17610. [PubMed: 21412425]

52. Takahashi K, Okita K, Nakagawa M, Yamanaka S. Induction of pluripotent stem cells from fibroblast cultures. Nat Protoc. 2007; 2:3081-3089. [PubMed: 18079707]

53. Prockop DJ, Kota DJ, Bazhanov N, Reger RL. Evolving paradigms for repair of tissues by adult stem/progenitor cells (MSCs). J Cell Mol Med. 2010; 14:2190-2199. [PubMed: 20716123]

54. Caplan AI, Correa D. The MSC: an injury drugstore. Cell Stem Cell. 2011; 9:11-15. [PubMed: 21726829]

55. Friedenstein AJ, Chailakhyan RK, Latsinik NV, Panasyuk AF, Keiliss-Borok IV. Stromal cells responsible for transferring the microenvironment of the hemopoietic tissues. Cloning in vitro and retransplantation in vivo. Transplantation. 1974; 17:331-340. [PubMed: 4150881]

56. Au P, Tam J, Fukumura D, Jain RK. Bone marrow-derived mesenchymal stem cells facilitate engineering of long-lasting functional vasculature. Blood. 2008; 111:4551-4558. [PubMed: 18256324]

57. Melero-Martin JM, et al. Engineering robust and functional vascular networks in vivo with human adult and cord blood-derived progenitor cells. Circ Res. 2008; 103:194-202. [PubMed: 18556575]

58. Moioli EK, et al. Synergistic actions of hematopoietic and mesenchymal stem/progenitor cells in vascularizing bioengineered tissues. PLoS One. 2008; 3:e3922. [PubMed: 19081793]

59. Park D, Sykes DB, Scadden DT. The hematopoietic stem cell niche. Front Biosci. 2012; 17:30-39.

60. Haniffa MA, Collin MP, Buckley CD, Dazzi F. Mesenchymal stem cells: the fibroblasts' new clothes? Haematologica. 2009; 94:258-263. [PubMed: 19109217]

61. Schrepfer S, et al. Stem cell transplantation: the lung barrier. Transplant Proc. 2007; 39:573-576. [PubMed: 17362785]

62. Lee RH, et al. Intravenous hMSCs improve myocardial infarction in mice because cells embolized in lung are activated to secrete the anti-inflammatory protein TSG-6. Cell Stem Cell. 2009; 5:5463. [PubMed: 19570514]

63. Kuznetsov, S.; Riminucci, M.; Robey, PG.; Bianco, P. Post-natal skeletal stem cells: methods for isolation and analysis of bone marrow stromal cells (BMSCs) from post-natal murine and human marrow. In: Celis, JE., editor. Cell Biology: A Laboratory Handbook. Elsevier; San Diego, CA: 2006. p. 79-86. 


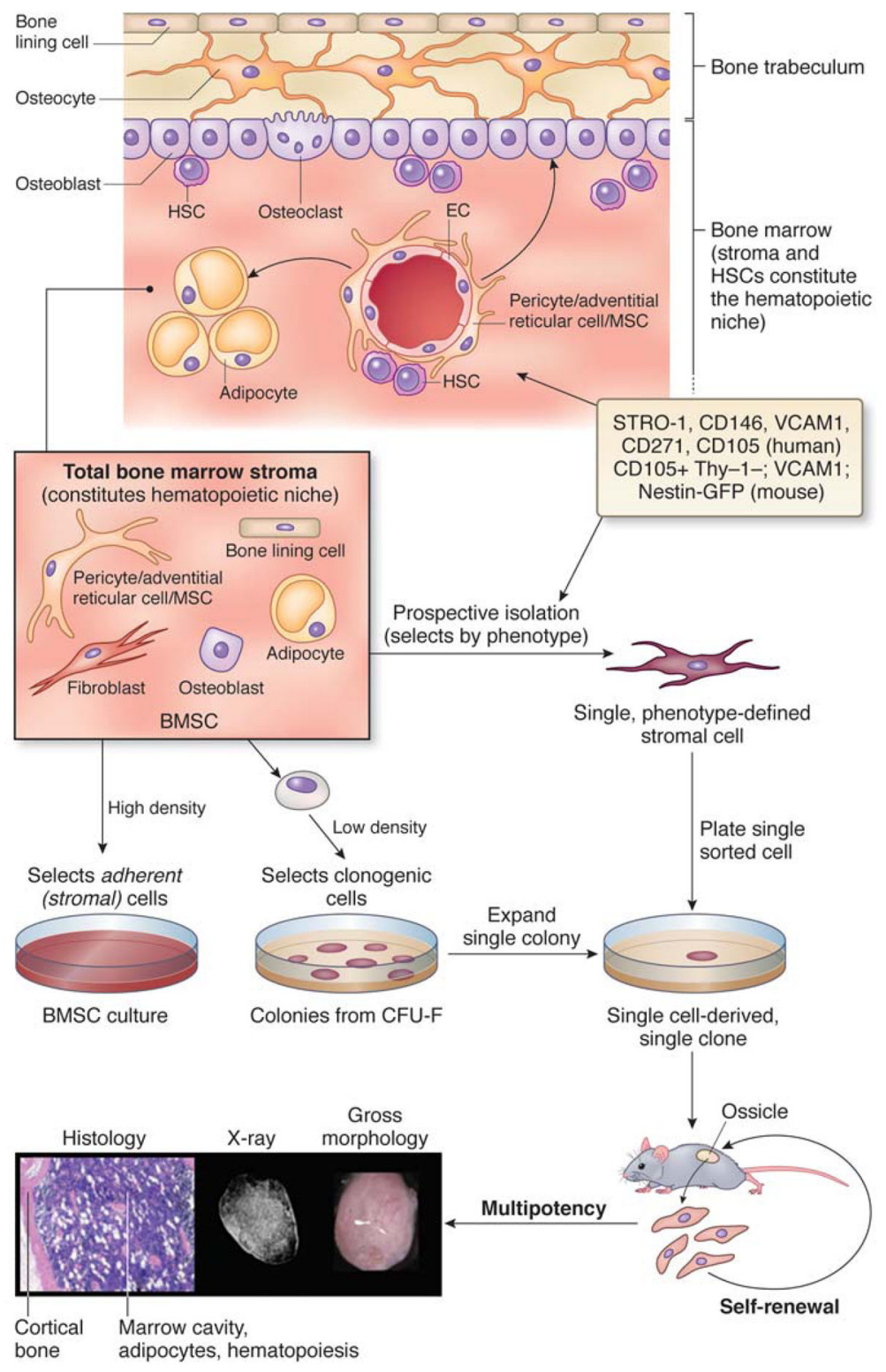

Figure 1. The whereabouts of MSCs

In the postnatal BM, 'MSCs' reside around sinusoids, maintain a 'niche' for HSCs, support hematopoiesis, and replenish the differentiated compartment of osteoblasts and adipocytes during tissue growth and turnover; they also generate cartilage under specific conditions such as trauma). Total populations of bone marrow stromal cells (which do not coincide with cultures of 'stem cells' in any way) are established when total marrow cell suspensions are plated in culture at high density. Adherence to plastic separates the stromal cells from no-adherent hematopoietic cells. If cell suspensions are plated a low density, only cells capable of density-insensitive growth are selected. These cells initiate the growth of clonal colonies. Passaging a single colony, or alternatively, establishing a clonal culture from single cells isolated based on expression of phenotypic markers (which may

be different in humans and mouse), results in a clonal population, which can be used to assess multipotency by in vivo transplantation. Heterotopic ossicles include multiple differentiated cell types of donor origin (osteoblasts, adipocytes, fibroblasts) and hematopoietic cells of the host. These are supported by donor stromal cells. X-ray and histology images show a heterotopic ossicle made by murine MSCs transplanted on a completely resorbable scaffold (GelfoamTM). 


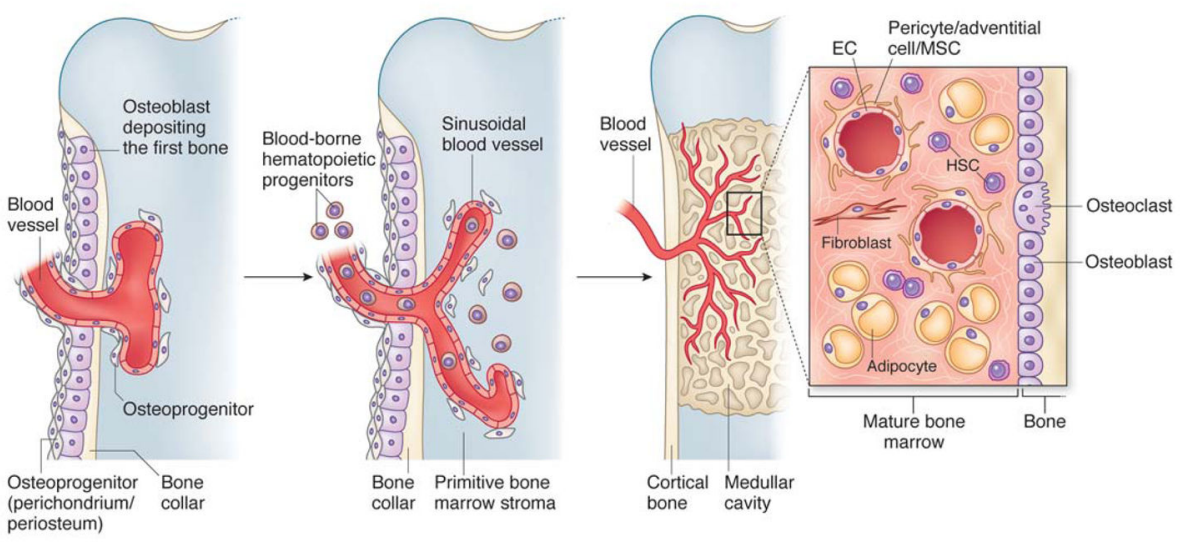

Figure 2. How MSCs make it to bone marrow

In development, the primitive bone marrow stroma include skeletal progenitors that originate outside of the marrow cavity (primitive periosteum-perichondrium) and invade the forming cavity along blood vessels. Similar dynamic interactions with ingrowing blood vessels are reproduced in transplants of human MSCs, and are likely the basis for the perisinusoidal position of

MSCs in the intact postnatal bone marrow. Recruitment of mesenchymal cells to a mural cell fate (and a subendothelial position), a general phenomenon in development and organ growth, is mediated by endothelial cell-derived PDGF-BB, which signals through PDGF-R $\beta$ expressed on mesenchymal cells (and on 'MSCs'). Presumptive mural cells produce Angiopoietin-1, (also produced by human and murine bone marrow MSCs2), which is crucial for integrity, survival and remodeling of vascular lattices. Ang-1 also induces quiescence of HSCs. Both mural cells and endothelial cell are induced to mitotic quiescence by active TGF 1, which is released through proteolytic cleavage of the latent form at sites of mural cell-endothelial cell contacts (reviewed in 4, 16, 38-40). 